\title{
Ebola Economics: The Case for an Upstream Approach to Disease Emergence
}

This month brings the cautious, hopeful news of an ebb in Ebola cases in West Africa. More than a year in, the heroic, selfless work of those battling on the front lines appears to be reining in a raging epidemic. But what can be said of global capacity to detect and control similar events at their inception? Are we prepared for-and is it possible to prevent $-\mathrm{a}$ future pandemic?

Like most pandemics, the current Ebola outbreak likely began with a single human-animal contact event, possibly in the index case, a 2-year-old boy who fell ill and died in December of 2013 in southeastern Guinea. Undetected, the virus moved out, carried by infected family members and care givers along rutted dirt tracks to neighboring communities, slipping quickly beyond the capacity of war ravaged and beleaguered public health systems to contain.

A series of events fanned the embers of this catastrophe-the inexorable churning of viral genetic code; altered landscapes facilitating contact and spillover from animal to human; evasion of detection and movement of the virus across travel networks to crowded urban centers-breathing life into a sputtering epidemic. Pandemics seem to share this playbook. Studying it yields three key insights on our collective preparedness.

First, our surveillance and front line disease detection is insufficient. Through 2012, Centers for Disease Control and Prevention-endorsed trained epidemiologists in West Africa ranged from 0.22 to 1.54 per million population, at best, only a third of CDC's recommended level. No trainees were produced in Liberia—or Guinea, where the outbreak originated. We must redouble our efforts to train and equip cadres of field epidemiologists who serve as the primary defense against these threats. These disease detectives should represent both human and animal health sectors, working collaboratively_and, critically, informed by community perspectives, cultural contexts, and appropriate communication strategies-to identify, rapidly diagnose, and respond to events. They must be capable of operationalizing those foundational principles-case finding, isolating sick patients, contact tracing, quarantine-that can shift the course of events, tipping the balance toward snuffing out a cluster at its inception.

Second, laboratory diagnostic capacity in the most risk-prone regions is lacking. This strain of Ebola virus had not previously been diagnosed in West Africa, yet compelling evidence suggests it was circulating there in an unknown animal population for the last decade. Tethered to boots-on-the-ground field epidemiologists must be capacity to rapidly produce accurate diagnostic results, facilitating arrival at a definitive diagnosis and informing response.

Third, our defensive posture is no longer adequate. Modernity demands a rebalance from our historically reactive, emergency control and containment to a proactive, preventative approach. This is not a radically new concept for medicine-prevention is the basis of modern public health, but the scale is much larger, and solutions will need to be innovative and imaginative. While it's unlikely we will ever definitively know how this virus moved out from the forest-that fateful spillover event that set the chain of transmission alight-we can be certain that these events are not aberrations. Indeed, they are representative of a new normal against which we must recalibrate our international preparedness and response.

A sound body of evidence has documented the rapidly expanding pace of disease emergence events in recent decades. If it seems as though pandemic threats are in- 
creasingly dominating headlines, it is, in fact, because they are. The public health community is watching closely the Middle East Respiratory Syndrome (MERS) coronavirus in the Arabian Peninsula, and is preparing for a recrudescence of H7N9 influenza in China this winter. What binds these diseases together is the human hand in their emergence. Most emerging diseases originate in animals, and their emergence is driven by our expansion of road networks, land use change, our intensification of livestock production, bushmeat hunting, and the wildlife trade. A human population approaching 9 billion by 2050 is etching an indelible mark on the planet. How we produce food and extract resources to fuel economic growth, compounded by aggressive land development and a changing climate are driving human and animal populations into unprecedented levels of contact. These are the seeds of a future pandemic.

This new landscape of risk challenges the very way in which we think about protecting global public health and mitigating the social and economic instability these pandemic threats provoke. While the preventative approach to pandemics seems like a daunting task, there are simple steps we can take.

Pandemics tend to emerge in regions with dense human populations, rapid development, and high wildlife biodiversity-regions known as emerging disease hotspots. Targeting prevention and surveillance programs geographically to these regions reduces the size of the funding burden. Further, programs that help reduce high-risk human activities in these regions can be effective at preventing the first spillover of a pathogen from wildlife to people. Efforts to alter wildlife market chains, slaughtering and butchering practices, hunting of high-risk species, and other behavior that brings people into close contact with zoonoses can be successful mitigation strategies. And pushing upstream in addressing disease emergence should be complemented by policy that views these events through an economic lens.

The World Bank estimates direct economic impact from the Ebola epidemic on the three most affected countries in West Africa through 2015 at $\$ 1.6$ billion-more than $12 \%$ of their combined GDP. If the epidemic is not brought under control, economic impact across Africa in 2015 could reach $\$ 6$ billion. While this upper estimate is unlikely under conditions of waning incidence, undoubtedly the path to pre-outbreak levels of growth and investor confidence will be arduous. Already, this Ebola virus event is the lengthiest in recorded history and has accounted for over ten times the number of cases than all previous known outbreaks combined. Borders have been closed, states of emergency declared, and travel and trade in the region severely constrained. The FAO has warned of severe food shortages. Previously, the World Bank placed the cost of six zoonotic disease outbreaks-diseases such as SARS and avian influenza transmissible from animals to humansfrom 1997 to 2009 at $\$ 80$ billion, an annualized burden of $\$ 6.7$ billion. Yet pre-Ebola outbreak levels of pandemic prevention funding amounted to a fraction of these costs, following an economic downturn that drastically contracted budgets for global disease detection and response. This defies logic that should prioritize more cost effective investments in prevention and early detection over the much larger expenditures needed for control and containment.

Solutions that identify the economic benefits of alternative, lower-risk activities could provide an answer. These solutions will need to be imaginative and daring. Instead of logging pristine forest to grow crops, what about incentivizing use of already-converted land? The economic costs and benefits of these strategies need to be examined, but above all the cost of emerging diseases needs to be incorporated in decisions on land use policy and development.

In September, the White House hosted a Global Health Security Agenda meeting, convening over 40 nations in commitments to accelerate activities toward the prevention, detection, and response to infectious disease threats. Such high profile fora help sustain the vigilance, political commitment, and resources required to achieve a shared vision for a world free from pandemic threats. Ebola virus in West Africa, much like the $2009 \mathrm{H} 1 \mathrm{~N} 1$ pandemic influenza, serves as a shot across the bow. We must take the dual path of addressing upstream emergence risk while ensuring systems are capable of finding that 2-year-old child, rapidly diagnosing and halting an epidemic in its tracks. Not to utilize these events as test preparation is an opportunity we can ill afford to miss.

\section{Daniel Schar}

U.S. Agency for International Development, Bangkok, Thailand

e-mail: dschar@usaid.gov

Peter Daszak

EcoHealth Alliance, New York, USA

Published online: February 18, 2015 